\title{
RHEUMATIC MANIFESTATIONS OF SCURVY: A CASE-REPORT
}

Clarice Moura Mata Machado ${ }^{1, \star}$, Matheus Fonseca Cardoso ${ }^{1}$, Carolina Ruas Freire Santos ${ }^{1}$, Lucas Moysés Carvalho de Oliveira ${ }^{1}$, Anna Carolina Faria Moreira Gomes Tavares ${ }^{1}$, Maria Fernanda Brandão de Resende Guimarães ${ }^{1}$

1.Universidade Federal de Minas Gerais, Belo Horizonte (MG), Brazil.

*Corresponding author: claricematamachado@gmail.com

\section{BACKGROUND}

Scurvy is a rare disease, caused by a deficiency of vitamin C, which is found in some fruits and vegetables. It can develop in individuals living in poverty or social isolation and with psychiatric disorders. Ascorbic acid, a reduced form of vitamin C, in addition to its antioxidant capacity, is an enzymatic cofactor necessary for collagen synthesis. Therefore, its deficiency can affect the synthesis of constituents of vessel walls and connective tissue. In addition to musculoskeletal manifestations, scurvy can also manifest with constitutional symptoms, skin and mucosal findings, and hemorrhagic manifestations.

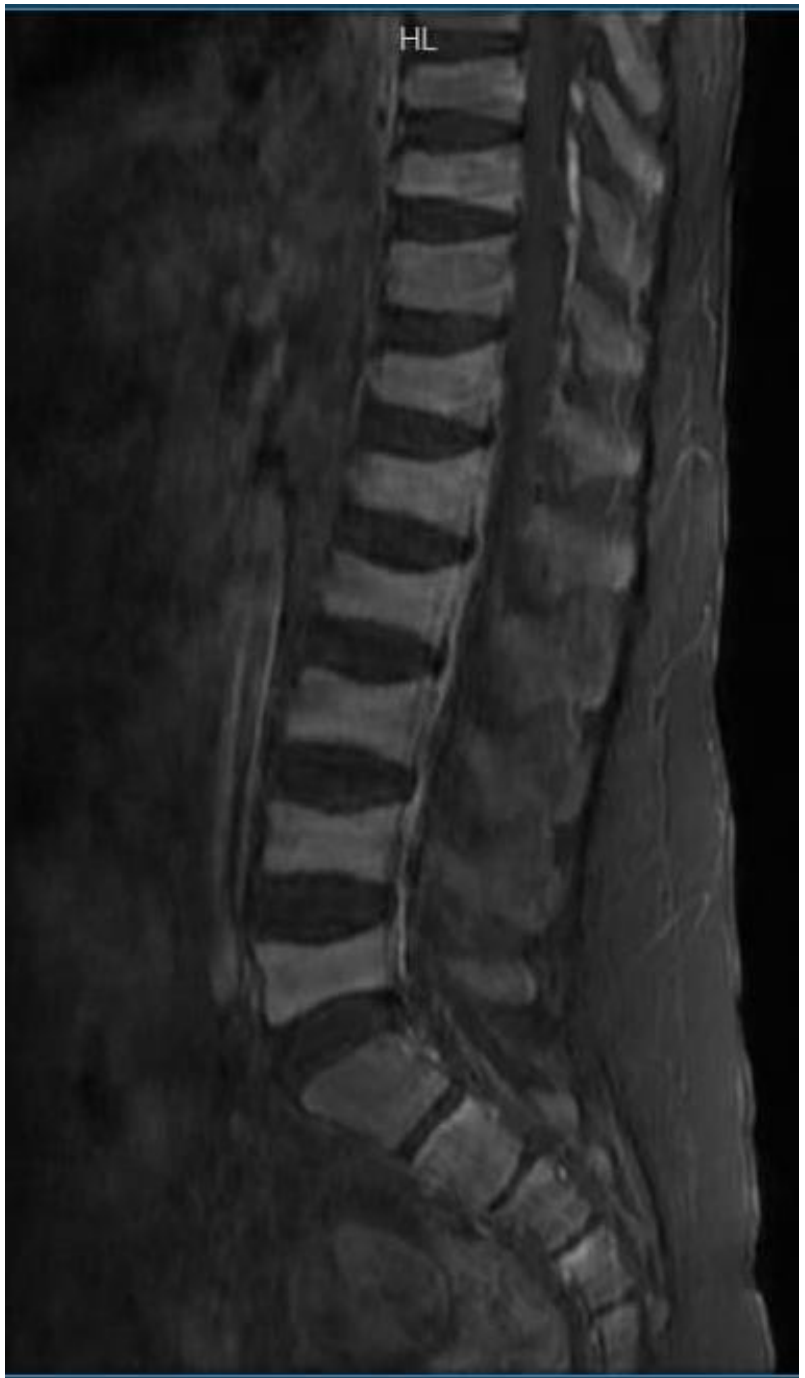

Figure 1. Sagittal T1 weighted image of the spine showing accentuated concavity of the plateaus of vertebral bodies. 


\section{CASE REPORT}

This is a 9-year-old male child, obese, with a 4-month history of weight loss, myalgia, inflammatory low back pain and prolonged morning stiffness, in addition to disabling pain in the lower limbs, ankles and in the Achilles tendon, perifollicular purpura with hyperkeratosis, gingival hypertrophy and hyperchromia and productive cough. In family history, the father had psoriasis. On physical examination, he had arthritis in his left ankle and knee, as well as pain on palpation of the entheses. Laboratory tests showed microcytic and hypochromic anemia and neutropenia. Magnetic resonance imaging (MRI) of the spine revealed accentuated concavity of the plateaus of some vertebral bodies (Figure 1), in addition to contrast impregnation in the spinous process of C7 and transverse processes from $\mathrm{T} 7$ to $\mathrm{T} 10$ on the left, along the costotransverse joints, as well as bordering the sacral vertebral plateaus and the visible portion of the sacroiliac joint. Foot and ankle MRI showed edema in the posterior apophysis of the calcaneus, osteopenia, small posterior tibiotalar and subtalar joint effusion, mild peri-insertional tendinopathy of the Achilles tendon, with paratendinitis. These findings raised the suspicion of a collagen disease. Chest tomography showed a tree-in-bud pattern and ground-glass opacities, which were later associated with capillary fragility. Biopsy of skin lesions revealed perivascular and perifollicular lymphohistiocytic inflammatory infiltrate, in addition to perifollicular hemorrhage and hemosiderin deposits, findings consistent with those described in scurvy. Later, it was found that the child's diet was based only on rice and beans. He had a significant improvement with vitamin Creplacement.

\section{CONCLUSION}

The rarity of scurvy and its wide range of clinical manifestations can make diagnosis challenging. This nutritional disorder can mimic rheumatic diseases, especially when it manifests with lesions that resemble cutaneous vasculitis and joint effusions simulating arthritis.

\section{KEYWORDS}

Scurvy, Vitamin C, Arthritis. 